\title{
Primary Osteosarcoma of the Breast- A Case Report and Review of Literature
}

\author{
Seshikanth Middela • Melville Jones • William Maxwell
}

Received: 24 January 2009 / Accepted: 18 March 2009/Published online: 19 May 2011

(C) Association of Surgeons of India 2011

\begin{abstract}
Primary Osteosarcoma of the breast is an aggressive and relatively rare tumour whose prognosis is very poor.It is often a diagnostic dilemma to the pathologists and the surgeons are perplexed due to its aggressive behaviour as its pathology and prognostic factors are unclear. Due to its rarity we suggest pooling of tissue samples from various centres in biobanks for future studies (including molecular biological studies) so that its behaviour can be better understood.
\end{abstract}

Keywords Breast . Cancer. Osteosarcoma

\section{Introduction}

Primary osteosarcoma of the breast is a rare and aggressive tumor with a poor prognosis. Fewer than 150 cases have been described in the literature over the past 50 years [1]. Many of the cases are described in association with a biphasic tumor i.e fibro adenoma, phylloides tumor or carcinosarcoma [2]. We describe a case report of a patient who presented with a lump in her right breast which proved to be primary osteosarcoma of the breast.

S. Middela $(\triangle) \cdot$ M. Jones $\cdot$ W. Maxwell

Withybush General Hospital,

Haverfordwest,

Pembrokeshire, Wales, UK

e-mail:seshi@gmx.com

\section{Case Report}

A 69 year old, with no significant past history presented with a lump in the right breast, which she noticed two weeks earlier with no other significant symptoms. Examination revealed a three centimeters painless firm lump, confirmed as malignancy on fine needle aspiration cytology (FNAC).

A mammogram revealed a $4 \mathrm{~cm}$ lesion suggestive of malignancy. A wide local excision and axillary node sampling was performed. The specimen was a well defined $4 \mathrm{~cm}$ lobulated gritty mass. Microscopical sections showed malignant multinucleated polygonal giant cells. The intervening eosinophilic material showed presence of osteoid. There was no ductal carcinoma in situ or malignant phylloides tumor. Immunocytochemistry for broad spectrum cytokeratin, EMA, Cam 5.2, S100, alpha 1-antitrypsin, CD68, and Oestrogen receptor were performed. The cytokeratin marker and Cam 5.2 showed a strong positivity in surrounding breast structures and negativity for the tumor cells suggesting that this is a true sarcoma and not metaplastic in origin. It was ER negative. There was no evidence of any vascular invasion with tumor free margins. A diagnosis of Primary Osteosarcoma of the breast was made.

The sampled nodes did not show the presence of malignancy. The chest X-ray was clear. After Multidisciplinary discussion the patient underwent a total mastectomy for clearance which did not show evidence of any residual malignancy or no evidence of connection to the underlying skeleton. A chest $\mathrm{x}$-ray during her first follow up visit three 
months later showed bilateral pulmonary metastasis with left pleural effusion later confirmed on $\mathrm{CT}$ scan and bone scan with no liver metastasis though the patient herself was asymptomatic. The patient wished palliative care and died soon after.

\section{Discussion}

The case report exemplifies an aggressive behavior of a Primary osteosarcoma. The true incidence of the primary breast sarcomas is difficult to estimate. It is less than $0.1 \%[1-3]$ of all primary breast malignancies. The annual incidence of primary breast sarcoma is estimated to be around 45 new cases per 10 million women [4]. Presentation is usually at an advanced age in contrast with skeletal osteosarcomas where the patients are younger $[2,5]$. There are reports of osteosarcoma being diagnosed at the age of 96 years [6] however the usual mean age at presentation in some series has been reported around 64 yrs (range 22-82 years) [2, 7].

The antecedent factors considered included previous local irradiation $(10 \%)$ and trauma $(6 \%)$ but none are well documented [2]. They are usually very large mobile irregular tumors with axillary lymph nodal involvement as a part of widespread disease [7] or as a direct local extension of the tumor in about $20 \%-28 \%$ of patients $[2,8]$.

It has been suggested to arise from mesenchymal cells de novo or transformation of a fibroadenoma or phylloides tumour $[1,6]$. Its association with fibroadenoma or phylloides tumor can be partly explained by the fact that the earlier series included various histopathology types due to a lack of consensus $[6,9]$.

A sarcoma of the breast has three important differential diagnosis namely

i. Carcinosarcoma often referred as metaplastic carcinoma [10]

ii. Secondaries from skeletal osteosarcoma

iii. Osteosarcoma arising from underlying skeleton

Metaplastic carcinomas can be diagnosed by carcinomatous component on $\mathrm{H} \& \mathrm{E}$ sections [3], more so ever they are immunoreactive for epithelial markers such as cytokeratin and CAM 5.2 which implies that they are epithelial and has to be treated as a primary breast carcinoma $[2,3]$. The specific morphologic features like leaf like architecture and stromal overgrowth excludes phylloides tumour [3]. Production of osteid and bone is the characteristic feature of extraosseous osteosarcoma however a direct connection between the underlying skeleton and metastasis from skeletal osteosarcoma must be ruled out. [6]
Mammography usually shows a well defined dense and coarsely calcifiedlesion with well defined margins and lobulated borders [11]. Fine needle aspiration and core needle biopsy can help but mineralized bone tissue may sometimes make procuring of tissue difficult $[12,13]$ and differentiating cytologic patterns may be difficult [6]. Ultrasound scan shows irregularly shaped mass with heterogenous echogencity and significant posterior shadowing due to bone formation [12]. It can also show areas of necrosis and has been documented in $10 \%$ of cases [11].

Skeletal Scintigraphy by technetium 99-diphosphonate which is a specific radionuclide marker of osteoid tumoral tissue suggestive of bone forming tumors can be used to rule out primary skeletal osteosarcoma with breast metastasis[14] or to detect metastases post operatively [11 and 15]. High level of serum alkaline phosphatase has been documented which suggests bone forming neoplasms and also correlates with high histochemical expression in the tumor and has been noted to decline following excision $[5,11]$ hence useful in follow up [15]. Serum alkaline phosphatase was not measured in our case.

Surgery is the mainstay as long as negative margins of 2 to $3 \mathrm{~cm}$ can be achieved [9] and often a simple mastectomy is needed to achieve complete local control and prevent local recurrences [2, 7]. Axillary lymph node dissection is indicated only in the presence of clinically involved nodes, as the tumours have a propensity to spread by blood stream unlike epithelial cancers of the breast [2]. The use of frozen section has been advocated [8]. Adjuvant chemotherapy may be of benefit especially in selected young patients with large tumors however its role is unclear as the data available is small $[2,5]$. Systemic adjuvant therapy or local radiotherapy has not been recommended in those who undergo complete excision with clear margins though they are still at risk of distant metastases [7].

Prognosis is directly proportional to the size of the tumours, the larger the size the poor the prognosis and decreased survival $[2,5]$. They are very aggressive tumours with early recurrences and haematogenous spread, most frequently to the lungs $(80 \%)$ as exemplified by our case report. Bony metastasis $(25 \%)$ and liver metastasis (17\%) are the next common sites $[2,5]$.

Silver SA [2] et al reported a 5-yr survival rate of 38\%. The overall recurrence rate for an extraskeletal osteosarcoma is about $43 \%$ at a median of $1 \mathrm{yr}$ [5]. In addition to size, grade and subtype [2] adequate local control is an important prognostic factor [7, 11]. However the lack of a large data makes it difficult to correlate between any other suggested prognostic factors (tumor size, grade, 
nodal status, histology and the extent of resection) and the prognosis. The variously reported survival rates in the literature could be explained by the different subtypes of the osteosarcoma [16]

\section{Conclusions}

The pathology of these aggressive tumours is poorly understood and hence the right treatment modality is unknown. With the advent of screening programmes for the detection of breast cancers we have no doubt that more cases will be picked up at an early stage leading to treatment dilemmas. In order to understand the molecular biology and thus its behaviour we suggest pooling of the specimens in biobanks so that they can be analysed. More research is needed to understand its pathology which may help us to treat complex cases in the future.
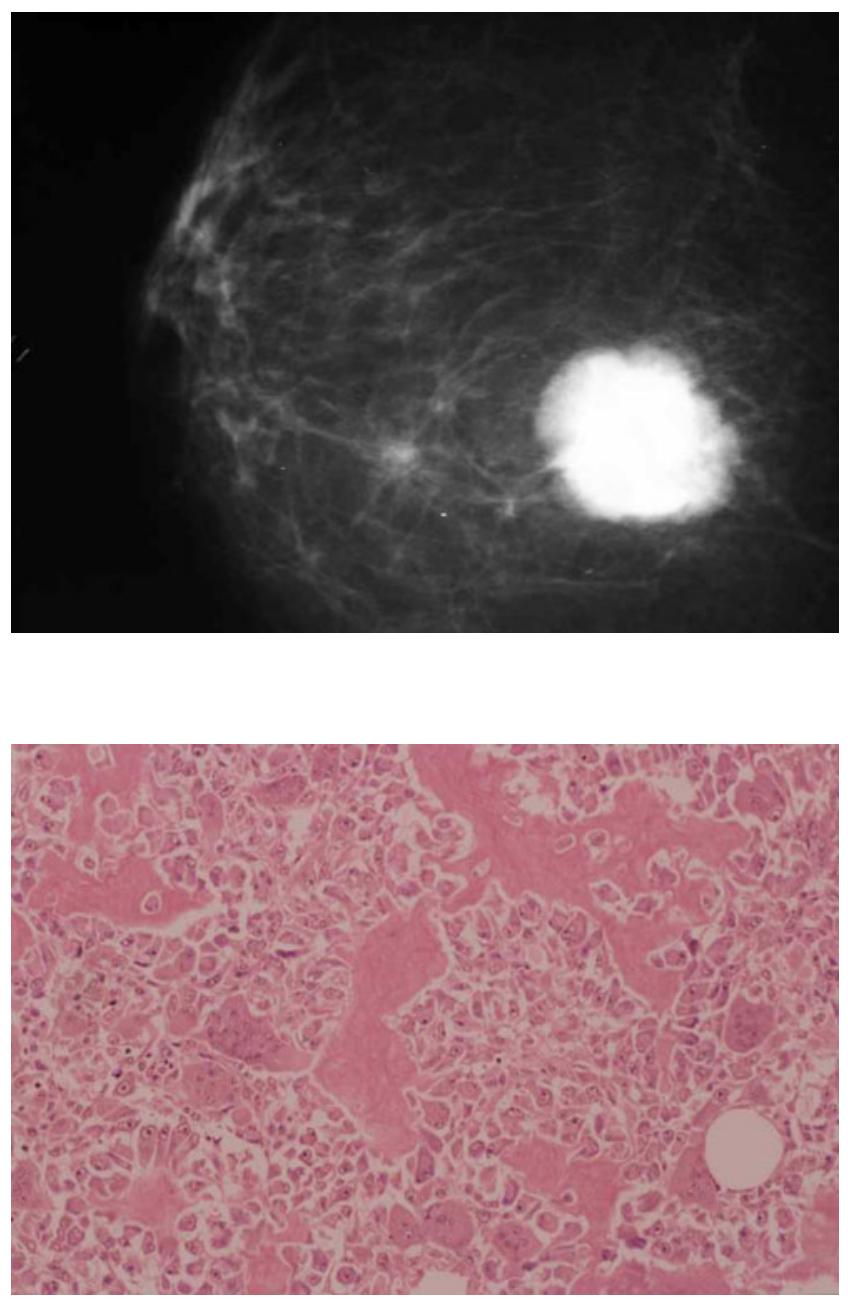

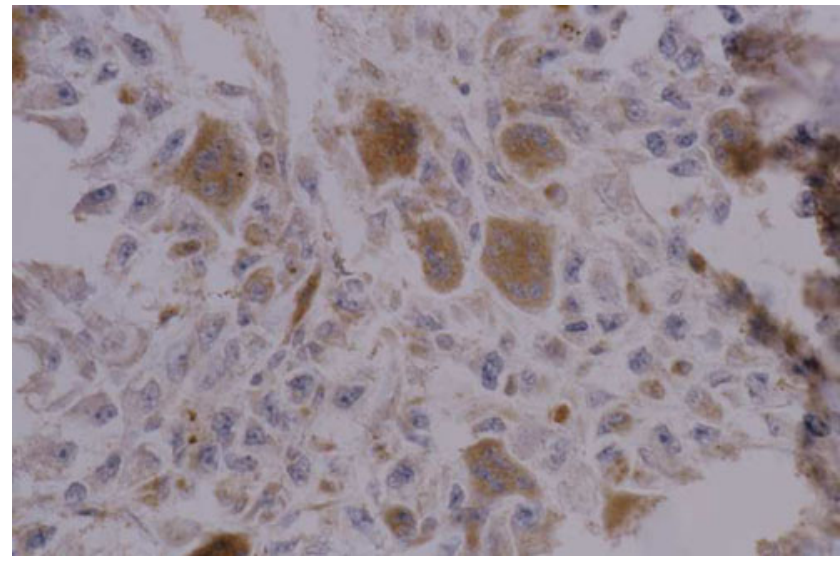

\section{References}

1. Khan S, Griffiths EA, Shah N et al (2008) Primary osteogenic sarcoma of the breast: a case report. Cases J 1(1):148

2. Silver SA, Tavassoli FA (1998) Primary osteogenic sarcoma of the breast: a clinicopathological analysis of 50 cases. Am J Surg Pathol 22(8):925-933

3. Adem C, Reynolds C, Ingle JN et al (2004) Primary breast sarcoma: clinicopathologic series from the Mayo Clinic and review of the literature. Br J Cancer 91(2):237-241

4. Confavreux C, Lurkin A, Mitton N et al (2006) Sarcomas and malignant phyllodes tumours of the breast-a retrospective study. Eur J Cancer 42(16):2715-2721

5. Vorobiof G, Hariparsad G, Freinkel W et al (2003) Primary Osteosarcoma of the breast- a case report. Breast J 9(3):231-233

6. Bahrami A, Resetkova E, Ro JY et al (2007) Primary osteosarcoma of the breast: report of 2 cases. Arch Pathol Lab Med 131 (5):792-795

7. Blanchard DK, Reynolds CA, Grant CS et al (2003) Primary Nonphylloides breast sarcomas. Am J Surg 186:359-361

8. Sabaté JM, Gomez A, Torrubia S et al (2002) Osteosarcoma of the breast. AJR 179:277-278

9. Lum WY, Jacobs L (2008) Primary breast sarcoma. Surg Clin North Am 88(3):559-570

10. Esses KM, Hagmaier RM, Blanchard SA et al (2009) Carcinosarcoma of the breast: two case reports and review of the literature. Cases J 2(1):15

11. Elemenoglou J, Zizi-Serbetzoglou A, Papatheofanis J et al (1999) Primary osteogenic sarcoma of the breast. A case report. Eur J Gynaecol Oncol 20(1):67-68

12. Mcdermott JP, Vorenberg A (2003) Differentiating osteogenic sarcoma from calcifying fibroadenoma. Contemp Surg 59(12):574-576

13. Muller AG, Van Zyl JA (1993) Primary osteosarcoma of the breast. J Surg Oncol 52:135-136

14. Yang JG, Li CL, Hao RR (2008) Primary osteogenic sarcoma of breast detected on Tc-99 m MIBI scintigraphy and Tc-99 m MDP skeletal scintigraphy. Ann Nucl Med 22(1):79-82

15. Brown AL, Holwill SD, Thomas VA et al (1998) Case report: primary osteosarcoma of the breast: imaging and histological features. Clin Radiol 53:920-922

16. Murakami S, Isozaki H, Shou T et al (2009) Primary osteosarcoma of the breast. Pathol Int 59(2):111-115 